Merma-Molina, G., Ávalos Ramos, M.A. y Martínez Ruiz, M.A. (2019). ¿Por qué no son eficaces los planes de convivencia escolar en España? Revista de Investigación Educativa, 37(2), 561-579.

DOI: http://dx.doi.org/10.6018/rie.37.2.313561

\title{
¿Por qué no son eficaces los planes de convivencia escolar en España?
}

\section{Why are school coexistence plans not effective in Spain?}

\author{
Gladys Merma-Molina, María Alejandra Ávalos Ramos y María Ángeles Martínez Ruiz \\ Departamento de Didáctica General y Didácticas Específicas. Universidad de Alicante (España)
}

\begin{abstract}
Resumen
Uno de los grandes problemas de la educación española es el incremento de la conflictividad y de la violencia dentro y fuera de los centros escolares. Existen programas de prevención e intervención puestos en marcha por la administración educativa, como los planes de convivencia, que no han tenido los resultados esperados. El objetivo de este estudio es analizar y evaluar los planes de convivencia que se están implementando en centros de Educación Secundaria en España. Para ello se ha tomado una muestra de $N=806$ centros educativos españoles. Se utilizó la Matriz de evaluación de los planes de convivencia validada a través del juicio de expertos; las valoraciones de los items seleccionados se han operativizado con el Método de Osterlind (1989). La fiabilidad del instrumento obtuvo un Alpha de Cronbach de.876 de consistencia; los análisis estadísticos de los datos se realizan con el programa SPSS 23.0. Para la evaluación de los Planes de Convivencia se empleó la metodología participativa (De Miguel, 2009), donde intervinieron 5 expertos y 30 estudiantes-investigadores. Los hallazgos evidencian que la única fortaleza de los planes de convivencia es el adecuado planteamiento de los objetivos, mientras que las debilidades son el diagnóstico, que no refleja la realidad personal, familiar ni el entorno social del alumno; la inadecuada temporalización de las actividades; la falta de responsables y el deficiente seguimiento y evaluación. Se concluye que es necesario un enfoque holístico, práctico y realista de la convivencia en los centros orientado al cambio en la cultura escolar.

Palabras clave: violencia; educación para la paz; proyecto educativo del centro; prevención; plan.
\end{abstract}

Correspondencia: Gladys Merma Molina. gladys.merma@ua.es. Carretera de San Vicente del Raspeig s/n, 03690, Alicante. 


\begin{abstract}
One of the great problems of Spanish education is the increase of conflict and violence inside and outside schools. There are prevention and intervention programs put in place by the educational administration, such as coexistence plans, which have not had the expected results. The objective of this study is to analyse and evaluate the coexistence plans that are being implemented in Secondary Education centres in Spain. For this, a sample of $N=806$ Spanish educational centres has been taken. The evaluation matrix of coexistence plans was used, validated through expert judgment; the valuations of the selected items have been operationalized with the Osterlind Method (1989). The reliability of the instrument obtained an Alpha of Cronbach of.876 of consistency; the statistical analyses of the data are carried out with the SPSS 23.0 program. For the evaluation of the Coexistence Plans, the participative methodology was used (De Miguel, 2009) in which 5 experts and 30 student-researchers intervened. Findings show that the strength of the coexistence plans is the adequate setting of the objectives, while the weaknesses are the diagnosis, which does not reflect the personal, family or social environment of the student; inadequate timing of activities; the lack of responsible; and poor monitoring and evaluation. It is concluded that a holistic, practical and realistic approach for coexistence in schools is necessary, aimed at changing school culture.

Keywords: violence; education for peace; educational project of the center; prevention; plan.
\end{abstract}

\title{
Introducción
}

Uno de los mayores problemas que enfrenta la educación y la sociedad española es el incremento de factores disconvivenciales, especialmente de la disrupción, indisciplina, violencia de género, racismo, xenofobia, consumo de alcohol y acoso escolar (Gázquez, Pérez-Fuentes, Lucas \& Fernández, 2011; Musitu, Suárez, Del Moral \& Villareal, 2015; Piñero, Arense, López \& Torres, 2014). El creciente número de noticias reflejan esta peligrosa realidad: en Madrid, La Cadena Ser, el 14 de noviembre de 2016 entrevista a los padres de una niña que durante siete años sufrió acoso escolar. La historia de Carmen (nombre ficticio) comienza cuando cursaba $1^{\underline{0}}$ de primaria. Durante todo este tiempo es víctima de agresiones físicas y verbales; cuando los padres, impotentes de que no se hiciera nada en el centro, piden ayuda a la policía, el centro activa un protocolo que concluye que no existe bullying. Carmen actualmente sufre trastorno por estrés postraumático. El 10 de enero de 2017 una adolescente de trece años, en la Pedanía de Aljucer (Murcia), se ahorcó en su habitación; se sabe que sus compañeros la insultaban por su apariencia física. El 30 de enero de 2017 un estudiante de 17 años con un expediente académico brillante sembró el pánico en un Instituto de Villena (Alicante) hiriendo a cinco compañeros; las investigaciones posteriores concluyen que era víctima de acoso escolar. Lo que parece evidente, en los casos señalados, es que los protocolos en los centros educativos no se activaron o, si se implementaron, no fueron efectivos.

Existen múltiples protocolos de prevención e intervención, especialmente en casos de acoso escolar, que se han puesto en marcha como el Programa Aprender a ser persona y a convivir: un programa para secundaria (Trianes \& Fernández-Figarés, 2001), Sevilla antiviolencia escolar (Ortega, 1997a), Andalucía antiviolencia escolar (Ortega \& Del Rey, 
2001) o el Modelo Integrado (Torrego, 2006), así como los programas desarrollados por las administraciones públicas que se traducen en los planes de convivencia (en adelante PC). Estos proyectos, pese a ser propuestas interesantes, no han tenido los resultados esperados.

En España no se ha efectuado ningún estudio que evalúe la efectividad de los PC, pero se han realizado investigaciones que han valorado otras estrategias para mejorar la convivencia en los centros. Álvarez-García, Dobarro, Rodríguez Núñez y Álvarez (2013) analizan estas medidas y concluyen que hay una tendencia general a que el alumnado considere cada vez menos habituales la puesta en marcha de actuaciones para la mejora de la convivencia. Arguyen que hay una evolución hacia estrategias basadas en la imposición y difusión de normas y sanciones en detrimento de estrategias educativas como la resolución de conflictos, el consenso de las normas y la educación en valores. Esto es contrario a lo que cabría esperar pues son conocidos los beneficios de estas medidas en el clima del aula y del centro, y en el rendimiento académico (PérezFuentes, Álvarez-Bermejo, Molero, Gázquez \& Vicente, 2011). También Álvarez-García, Rodríguez, González-Castro, Núñez y Álvarez (2010), y Penalva, Hernández y Guerrero (2014) destacan la escasa importancia atribuida a la normativa sobre convivencia escolar, y Cerezo (2011) sostiene que los padres no son informados convenientemente sobre las acciones previstas en el PC. Últimamente, Conde, Azaustre y Delgado (2015), basándose en el modelo de la European Foundation Quality Magnagement, han analizado la convivencia en 46 centros educativos andaluces con el objetivo de describir la gestión de la convivencia, confirmando la validez del modelo.

A partir de esta problemática y a los antecedentes expuestos, el objetivo general de este estudio es analizar los PC que se están implementando en los centros de Educación Secundaria en España ${ }^{1}$. Los objetivos específicos son:

1) Analizar la presencia y la calidad de los elementos fundamentales de los PC.

2) Identificar las fortalezas y debilidades de los PC.

3) Proponer recomendaciones y estrategias de mejora en el diseño y desarrollo de los PC.

El incremento de la presencia de factores que afectan negativamente a la convivencia y consecuentemente al aprendizaje y que pone en duda la eficacia de los PC han motivado el planteamiento de las siguientes cuestiones de investigación:

¿Cómo se realiza y diseña el diagnóstico de los PC en los centros de Educación Secundaria?

¿Cómo se diseñan y están planteados los objetivos de los PC?

¿Cómo se ha planificado las actividades y qué tipo de actividades se prevén?

¿Cómo se ha realizado la planificación temporal de las actividades?,

¿Cómo se asignan a los responsables de las actividades previstas en el PC?

¿Cuáles son las fortalezas y debilidades de los PC?

1 Este estudio ha sido financiado por el Vicerrectorado de Calidad e Innovación Educativa de la Universidad de Alicante (Referencia 3806/BOUA-2016) en el marco del Proyecto de Redes-I3CE de investigación en Docencia Universitaria cuyo título es "La autoridad de profesor. Diagnóstico de la situación" y se ha desarrollado en el Grupo de Investigación Interdisciplinar en Indisciplina y Violencia Escolar de la misma universidad. 


\section{La convivencia y los planes de convivencia en España}

La investigación sobre la convivencia escolar tiene ya cierto recorrido. En los años noventa, Ortega (1997b) y Díaz-Aguado, Segura, Royo y Andrés (1996) empiezan a identificar el bullying; estos autores plantean líneas de investigación vinculadas con la agresión y el acoso entre iguales y proponen programas para mejorar dichas problemáticas. El término convivencia es empleado en el ámbito académico-educativo por Carbonell (1997); sin embargo, fue Jares (1997) quien enfoca la convivencia como un modelo transformador de la sociedad que se promueve en los centros educativos. Más adelante, Boqué (2002) añade el concepto cultura de paz como denominación de cualquier programa que fomente la mejora de la convivencia escolar y Torrego (2006) defiende la importancia de una normativa específica basada en medidas dialogantes. De esta manera, en el siglo XXI la convivencia ha pasado de ser un hecho requerido por instituciones educativas pioneras o sensibilizadas a un objetivo de primera línea que se incluye en los proyectos educativos de los centros (en adelante, PEC), creándose planes específicos para promoverla (Zaitegui, 2010).

En 2006, la Ley Orgánica de Educación (LOE) proyecta la convivencia como un objetivo educativo prioritario. En consecuencia, en las 17 comunidades se legislan nuevas Órdenes y Decretos para implementar los PC; así, en un periodo de tres años, prácticamente todos los centros escolares los incorporan en sus documentos administrativos y de gestión (Zaitegui, 2010). Posteriormente, la LOMCE (2013) reconoce explícitamente la necesidad de educar para la convivencia como base fundamental para lograr el éxito personal, social y académico del alumnado. La norma tiene en cuenta los principios de equidad, inclusión, igualdad de derechos y oportunidades, la eliminación de la discriminación, la prevención de conflictos y la resolución pacífica de los mismos, la no violencia, y la prevención del acoso escolar y de la violencia de género; pese a ello, los casos de violencia, bullying, agresividad, disrupción e indisciplina siguen siendo elevados, especialmente en primero y segundo de la ESO (Gil, Delgado \& Chillón, 2016), y los profesores se quejan de los incidentes violentos y de la falta de colaboración del alumnado y de sus familias (Cerezo, 2011). ¿A qué se debe esta problemática? Es probable que en este estudio encontremos algunas respuestas.

El PC se ha definido tradicionalmente como un programa que incluye medidas para la prevención e intervención de los problemas de convivencia que afectan al alumnado (Dobarro, García \& Álvarez, 2013) sin apenas incorporar medidas relativas a la mejora de la convivencia que involucre a toda la comunidad educativa. Tampoco se ha tenido en cuenta el carácter estratégico, integral, flexible y dinámico ni su coherencia con el PEC.

Por su naturaleza estratégica, el PC ha de partir del diagnóstico del centro que permita identificar los puntos débiles y fuertes vinculados con la convivencia (Conde, Azaustre \& Delgado, 2015), ofreciendo una visión real del profesorado, del alumnado, de las familias, del personal de administración y servicios, y del contexto donde se ubica del centro. Los objetivos, en coherencia con el diagnóstico, dan respuesta a las necesidades de la comunidad educativa, y el diseño de las acciones y su puesta en marcha responde al diagnóstico y a los objetivos priorizando la importancia de las actuaciones preventivas (Padrós, 2014) siendo crucial, además, la planificación temporal y la asignación de responsables. Finalmente, de forma periódica, es fundamental 
detenerse a valorar la eficacia del PC para, en su caso, actualizarlo o adaptarlo a las nuevas situaciones. El objetivo del seguimiento y evaluación del PC es analizar el impacto de las acciones, el logro de objetivos, la participación de todos los miembros de la comunidad educativa, los recursos utilizados, las acciones formativas, el asesoramiento técnico, la colaboración de instituciones locales y el grado de conocimiento del PC por la comunidad educativa.

\section{Método}

La investigación es de tipo explicativo-interpretativa. Para una mejor comprensión del estudio se utiliza el enfoque mixto -cuantitativo y cualitativo- que se engloba en un diseño ex post facto, no experimental-transversal (Sabariego, 2012). El enfoque cuantitativo permite analizar los 17 ítems del instrumento susceptibles de ser medidos, y el cualitativo, indagar en las narrativas de los participantes de forma inductiva.

\section{Muestra}

La selección de la muestra se llevó a cabo mediante el muestreo no probabilístico intencional cuyo criterio inicial fue el acceso a los planes de convivencia de los centros educativos españoles. La muestra estuvo constituida por 806 PC de Institutos de Educación Secundaria Obligatoria de las 17 Comunidades Autónomas de España y fue seleccionada, finalmente, considerando la disponibilidad de los PC en las direcciones web de los propios centros (Gráfico 1).

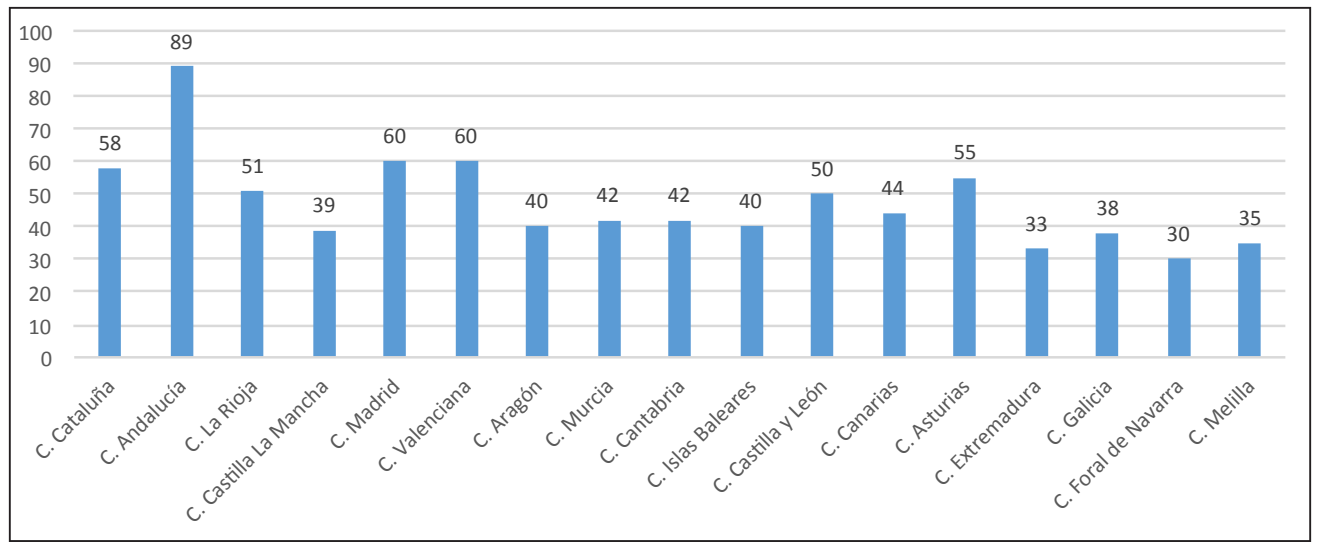

Gráfico 1. Porcentaje de la muestra por Comunidades Autónomas

\section{Instrumento}

Al no existir otros estudios similares, se diseñó un instrumento ad hoc, la Matriz de evaluación del Plan de convivencia (en adelante MPC). Se elaboró una primera versión constituida por 22 ítems teniendo como referencia otras investigaciones y la literatura 
relevante sobre el tema (Burton, 2012; Cerezo, 2011; Dobarro, García \& Álvarez, 2013; Ortega, Del Rey \& Casas, 2014; Torrego, 2006; Zaitegui, 2010).

El instrumento se validó a través del juicio de expertos (5 investigadores especialistas en el tema) (en adelante GIE), verificándose la pertinencia de los ítems en función de su correspondencia y adecuación con los componentes básicos del PC. Además, para cada ítem se proporcionó un espacio en la matriz para que el GIE incluya sus observaciones y/o recomendaciones. Con el fin de sintetizar de una manera operativa las valoraciones del GIE sobre la adecuación de los indicadores seleccionados, se utilizó el Método De Osterlind (1989) con una escala de tres niveles de congruencia (-1 si es baja, $0=$ si es media y 1 si es alta). 17 ítems alcanzaron una congruencia de Osterlind mayor a O.5; cada ítem tenía cinco posibles valoraciones, basadas en la Escala de Likert y con valores que iban desde 1 hasta 5 , siendo 1 la puntuación más baja y 5 la más alta: 2 ítems para el diagnóstico, 3 para los objetivos, 2 para las actividades, 1 para la temporalización, 1 para los responsables de las actividades, 1 para la evaluación y 7 ítems para los objetivos y actividades vinculadas con la prevención e intervención del bullying y ciberacoso, con las conductas que alteran la convivencia de forma grave y reincidente (insultos, amenazas, agresiones, peleas y/o vandalismo), con el maltrato infantil, con la violencia de género, con las agresiones hacia el profesorado y al personal de administración y servicios, y con situaciones de violencia fuera del centro educativo que impliquen a algún miembro del centro.

Una vez determinados los ítems del cuestionario, se realizó la prueba de confiabilidad que muestra la constancia y precisión de la medida. Para ello se utilizó el Alfa de Cronbach que dio como resultado un coeficiente de.876, concluyéndose que el instrumento de recogida de información es óptimo y fiable (tabla 1).

Tabla 1

Estadísticas de fiabilidad del total de elementos

\begin{tabular}{lcccc}
\hline & $\begin{array}{c}\text { Media } \\
\text { de escala }\end{array}$ & $\begin{array}{c}\text { Varianza } \\
\text { de escala }\end{array}$ & $\begin{array}{c}\text { Correlación } \\
\text { total }\end{array}$ & $\begin{array}{c}\text { Alpha de } \\
\text { Cronbach }\end{array}$ \\
\hline $\begin{array}{l}\text { El diagnóstico permite conocer la situación } \\
\text { de conflictividad y convivencia del centro. }\end{array}$ & 39.17 & 106.299 & .530 & .869 \\
$\begin{array}{l}\text { El diagnóstico posibilita conocer la } \\
\text { situación familiar, las características } \\
\text { del entorno social y los conflictos de la }\end{array}$ & 39.52 & 107.441 & .476 & .871 \\
$\begin{array}{l}\text { localidad donde se ubica el centro. } \\
\begin{array}{l}\text { Los objetivos incluyen a todos los } \\
\text { miembros de la comunidad educativa y } \\
\text { favorece la comunicación entre ellos. }\end{array}\end{array}$ & 38.94 & 106.152 & .626 & .865 \\
$\begin{array}{l}\text { Hay objetivos vinculados con la mediación } \\
\text { y de resolución pacífica de conflictos. }\end{array}$ & 39.05 & 104.201 & .622 & .865 \\
$\begin{array}{l}\text { Los objetivos son reales, alcanzables y } \\
\text { fáciles de entender. }\end{array}$ & 38.85 & 103.601 & .635 & .864 \\
$\begin{array}{l}\text { Las actividades previstas se plantean a } \\
\text { nivel de aula y de centro. }\end{array}$ & 38.80 & 107.131 & .554 & .868 \\
\hline
\end{tabular}




\begin{tabular}{|c|c|c|c|c|}
\hline & $\begin{array}{c}\text { Media } \\
\text { de escala }\end{array}$ & $\begin{array}{l}\text { Varianza } \\
\text { de escala }\end{array}$ & $\begin{array}{c}\text { Correlación } \\
\text { total }\end{array}$ & $\begin{array}{l}\text { Alpha de } \\
\text { Cronbach }\end{array}$ \\
\hline $\begin{array}{l}\text { Hay actividades de prevención y de } \\
\text { intervención. }\end{array}$ & 38.80 & 105.194 & .668 & .863 \\
\hline $\begin{array}{l}\text { Existe un calendario de aplicación o } \\
\text { temporalización de las actividades } \\
\text { propuestas en el plan. }\end{array}$ & 39.83 & 104.768 & .535 & .868 \\
\hline $\begin{array}{l}\text { Se sabe qué personas se van a } \\
\text { responsabilizar de la aplicación de cada } \\
\text { una de las medidas diseñadas. }\end{array}$ & 39.29 & 103.366 & .573 & .867 \\
\hline $\begin{array}{l}\text { Se realiza el seguimiento y la evaluación } \\
\text { del plan. }\end{array}$ & 39.48 & 107.972 & .395 & .875 \\
\hline $\begin{array}{l}\text { Hay objetivos y actividades vinculados } \\
\text { con la prevención del bullying y del } \\
\text { ciberacoso. }\end{array}$ & 40.00 & 109.375 & .426 & .873 \\
\hline $\begin{array}{l}\text { Hay objetivos y actividades vinculados } \\
\text { con la intervención ante el bullying y el } \\
\text { ciberacoso. }\end{array}$ & 40.03 & 109.280 & .451 & .872 \\
\hline $\begin{array}{l}\text { Hay objetivos y actividades vinculados } \\
\text { con las conductas que alteran la } \\
\text { convivencia de forma grave y reincidente } \\
\text { (insultos, amenazas, agresiones, peleas y/o } \\
\text { vandalismo). }\end{array}$ & 39.34 & 109.009 & .404 & .874 \\
\hline $\begin{array}{l}\text { Hay objetivos y actividades vinculados } \\
\text { con el maltrato infantil. }\end{array}$ & 40.86 & 112.902 & .498 & .871 \\
\hline $\begin{array}{l}\text { Hay objetivos y actividades ante la } \\
\text { violencia de género. }\end{array}$ & 40.48 & 112.410 & .346 & .875 \\
\hline $\begin{array}{l}\text { Hay objetivos y actividades ante las } \\
\text { agresiones hacia el profesorado y personal } \\
\text { de administración y servicios. }\end{array}$ & 40.45 & 109.251 & .464 & .871 \\
\hline $\begin{array}{l}\text { Hay objetivos y actividades vinculados } \\
\text { con las situaciones de violencia fuera del } \\
\text { centro que impliquen a algún miembro del } \\
\text { centro. }\end{array}$ & 40.60 & 111.088 & .501 & .870 \\
\hline
\end{tabular}

Adicionalmente, en la MPC se incluye un apartado de valoración cualitativa que permite plantear conclusiones sobre los elementos básicos y destacar las fortalezas y debilidades.

\section{Procedimiento}

Para la evaluación de los PC se utilizó la metodología participativa (De Miguel, 2009), cuyo principio básico es que en la evaluación de programas de convivencia es importante utilizar el conocimiento y la experiencia de todos los implicados. Como se adelantó, en el estudio participaron cinco profesores-investigadores expertos, que pertenecen al Grupo de Investigación Interdisciplinar en Indisciplina y Violencia Escolar y 30 estudiantes que cursan el último cuatrimestre del Máster de Profesorado de Educa- 
ción Secundaria, quienes intervinieron voluntariamente en el proyecto. La condición para ser estudiantes evaluadores de los PC (en adelante EPC) fue haber cursado y superado la asignatura Sociedad, Familia y Educación donde se analizaron, desde una perspectiva teórico-práctica, los factores disconvivenciales que afectan el clima de las instituciones educativas, las normativas sobre el PC y el diseño, elaboración y ejecución del PC.

Una vez seleccionados los EPC, siguiendo los criterios de la evaluación participativa (De Miguel, 2009), se efectuó un programa formativo constituido por cuatro módulos con el objetivo de que los EPC adquieran conocimientos y habilidades suficientes para analizar los PC de forma coherente y responsable:

Tabla 2

Programa de formación de los EPC

\begin{tabular}{ll}
\hline \multicolumn{1}{c}{ Módulos } & \multicolumn{1}{c}{ Contenidos } \\
\hline Módulo 1 & $\begin{array}{l}\text { Perspectiva teórico-práctica de los factores disconvenciales que afectan } \\
\text { al clima del aula (conflicto, violencia escolar, bullying, ciberbullying, } \\
\text { indisciplina, disrupción, absentismo, fracaso escolar, sexting, grooming). }\end{array}$ \\
Módulo 2 & $\begin{array}{l}\text { Análisis de la normativa sobre la convivencia y los planes de convivencia } \\
\text { en las Comunidades Autónomas de España. }\end{array}$ \\
Módulo 3 & Estructura y elementos de los planes de convivencia. \\
Módulo 4 & ¿Cómo evaluar los PC? \\
\hline
\end{tabular}

Posteriormente el GIE recogió y seleccionó los PC de las páginas webs de los centros educativos siguiendo los siguientes criterios: 1) que los centros fueran de Educación Secundaria, 2) que los PC estuvieran vigentes en los cursos académicos 2014-2015 o 2015-2016 y 3) que el PC fuera un documento completo y no solo un resumen o una publicación parcial. Inicialmente se obtuvo una primera muestra de 875 PC de los cuales quedaron excluidos 69 debido a que no cumplían con alguno de los criterios mencionados. Entre los meses de enero y marzo de 2017 se analizaron los PC utilizando la MPC validada por el GIE.

Con el fin de que la información fuera objetiva, razonada y que las valoraciones sean producto de la reflexión personal (De Miguel, 2009), en abril y julio de 2017 se realizaron tres talleres más dirigidos por el GIE donde participaron los EPC con el fin de compartir experiencias y/o para plantear dudas sobre el proceso de evaluación de los PC.

El GIE realizó una meta evaluación sobre el trabajo evaluativo-participativo (Stufflebeam, 2001) a través de un proceso de triangulación y revisión crítica de la información aportada por los EPC, lográndose un 95\% de coincidencias tanto cuantitativas como cualitativas. Posteriormente, se realizó el proceso de almacenamiento y organización de la información en una base de datos, y se procedió a los respectivos análisis estadísticos con el programa SPSS. v. 23.0. 


\section{Resultados}

Los resultados se explican en cuatro temáticas: 1) el diagnóstico 2) los objetivos, 3) las actividades, la temporalización y los responsables de las acciones previstas, y 4) la evaluación y seguimiento del plan.

\section{Temática I. Sobre el diagnóstico}

Los hallazgos muestran que en el $16.92 \%$ de los PC no se ha realizado el diagnóstico de la convivencia; en el $16.92 \%$ no refleja la realidad del centro vinculada con la convivencia, en el $27.69 \%$, describe solo algunos problemas vinculados con el mal comportamiento de los alumnos, y en el $38.47 \%$ se identifican las características del clima del centro, destacando sus problemáticas y necesidades (Gráfico 2).

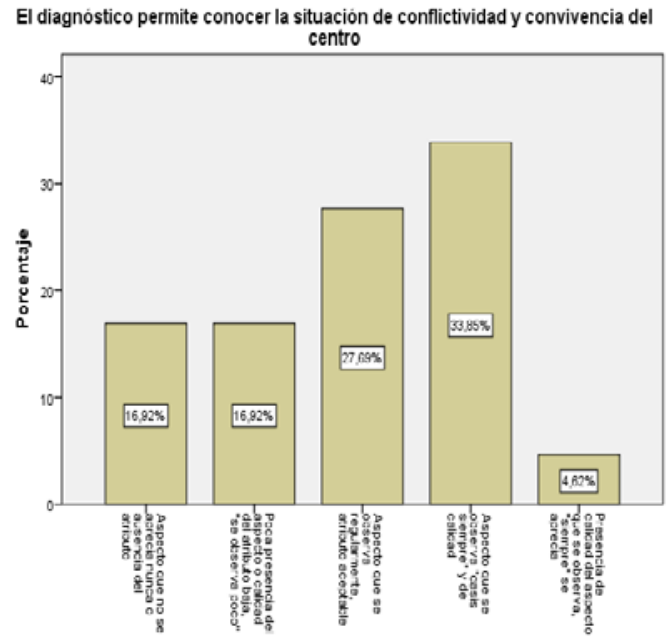

Gráfico 2. Diagnóstico de la convivencia del centro

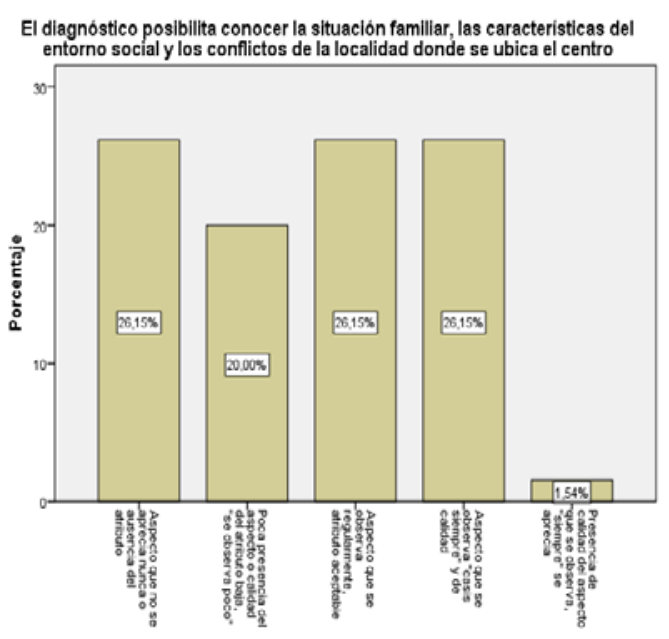

Gráfico 3. Diagnóstico de la convivencia de las familias y del entorno social

Un EPC señala al respecto:

“El PC analizado no tiene una contextualización del centro ni un diagnóstico sobre sus características ni las de su comunidad. Aunque en el documento los responsables sostienen que se ha hecho un diagnóstico previo, no hemos encontrado esta documentación en ningún lugar, por lo que no podemos reflexionar sobre si las medidas adoptadas son o no adecuadas y pertinentes, ni si tienen en cuenta todos las necesidades ni la problemática del centro. Partiendo de este punto, tenemos que hacer eco de la necesidad de tener un diagnóstico previo para poder llevar a cabo un PC que se adapte al IES" (EPC1Cat). 
En lo concerniente a si el PC refleja la situación de todos los miembros del centro que tengan una relación directa e indirecta con el alumnado (familias, profesores, personal de administración y servicios e instituciones del contexto), los hallazgos muestran que en el $26.15 \%$ de planes el diagnóstico no incluye el análisis de las familias, del profesorado, ni del entorno social donde se ubica el centro; el $20 \%$ ofrece una visión genérica de la realidad de las familias; en el $26.15 \%$ se puede conocer solo la realidad del alumnado y de sus familias; y únicamente en el $27.69 \%$ se reflejan los rasgos, problemáticas de los alumnos, la situación de las familias y el contexto social vinculados con la convivencia (Gráfico 3).

\section{Temática 2: Sobre los objetivos}

Los hallazgos muestran que en el $6.15 \%$ de PC no existen objetivos; en el $20 \%$, estos no se corresponden con el diagnóstico; en el $33.85 \%$ dan respuesta a la problemática y a las necesidades del centro vinculadas con la convivencia, y en el $40 \%$ se corresponden con el diagnóstico, incluyen a todos los miembros de la comunidad educativa y favorece la relación entre ellos (Gráfico 4).

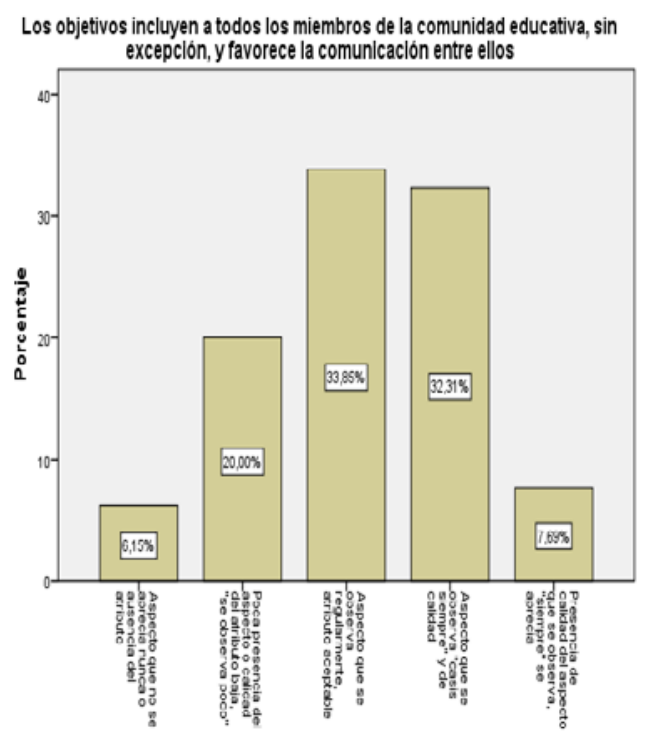

Gráfico 4. Alcance de los objetivos

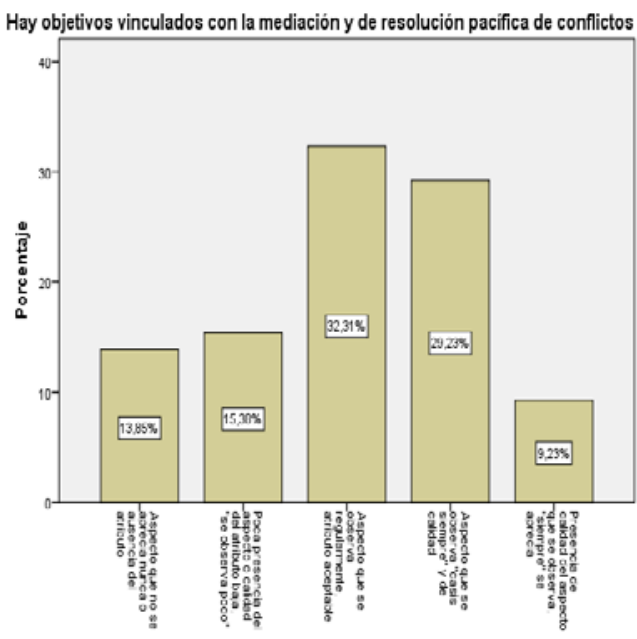

Gráfico 5. Objetivos de mediación y resolución de conflictos

La siguiente narrativa destaca este hecho:

“Se señala como objetivo general mejorar la convivencia en el instituto y luego otros objetivos específicos dirigidos a los alumnos, familias y a mejorar las relaciones entre todos los miembros de la comunidad educativa" (534Mel). 
Además, en el $13.85 \%$ de PC no existen objetivos vinculados con la mediación y resolución pacífica de conflictos; en el $15.38 \%$ se incluye algún objetivo de mediación de conflictos de manera muy general; en el 32.31\% los objetivos de este tipo son más específicos, pero involucra solo al profesorado, y el 38.46\% tienen objetivos de mediación y resolución de conflictos e implican al profesorado y al alumnado (Gráfico 5).

En lo concerniente a si los objetivos son realistas, factibles, concretos, claros, medibles, pertinentes y fáciles de entender, el 7.69\% de los PC carece de estos atributos; en el $23.08 \%$ son realistas; en el $21.54 \%$ son realistas y factibles, y en el $47.69 \%$ son realistas, precisos, claros, medibles, pertinentes y fáciles de entender (Gráfico 6).

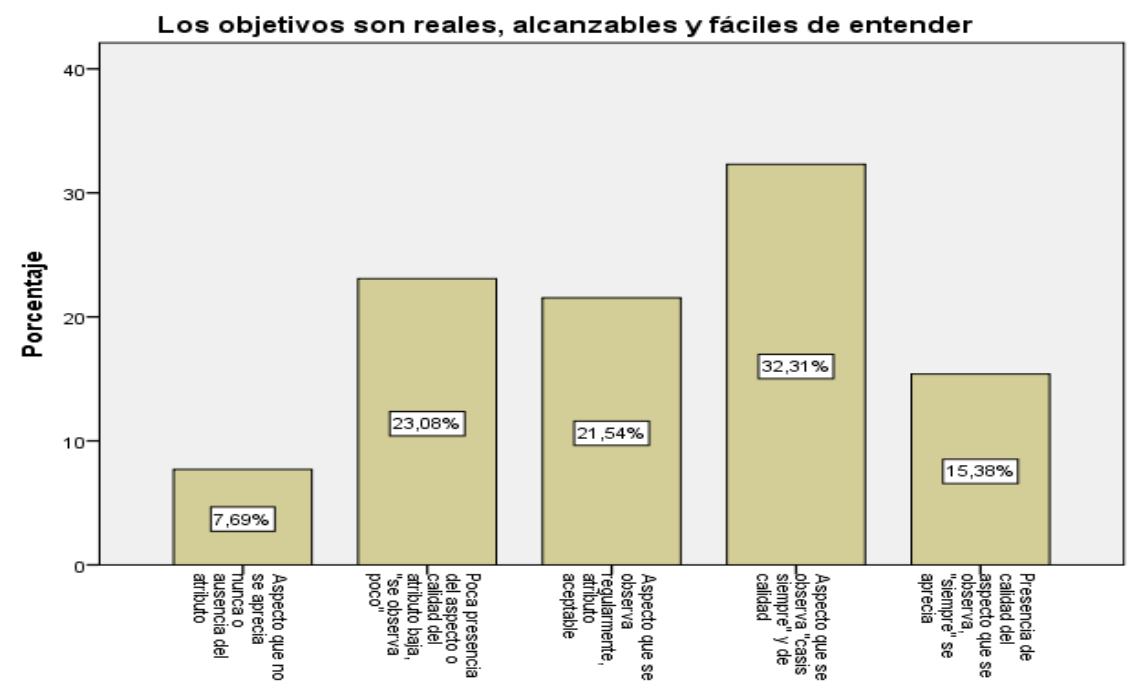

Gráfico 6. Calidad de los objetivos

Un EPC afirma:

"Se plantean objetivos generales reales y alcanzables como valorar y aprender el respeto a las normas; ayudar a los alumnos a formar una imagen ajustada de sí mismos y fomentar la colaboración entre el centro, la familia y la sociedad. Con relación a los objetivos específicos, han sido planteados para dar respuesta a la diversidad de alumnos o enseñar a "ser persona" y a convivir en armonía con el resto de la comunidad educativa" (59castillaleon).

\section{Temática 3. Sobre las actividades del plan de convivencia}

Los hallazgos muestran que en el $7.69 \%$ de los PC no se ha incluido ningún tipo de actividad a nivel de aula ni de centro; en el 10.77\% las acciones no se corresponden con los objetivos ni con el diagnóstico, ni se han previsto a nivel de centro ni de aula; en el $38.46 \%$ se relacionan parcialmente con los objetivos y con la realidad del centro y solo se han previsto a nivel de aula; y en el $43.08 \%$ el diseño de las actividades y su puesta en marcha responden al diagnóstico de la convivencia, a los 
objetivos previamente establecidos e incluyen actuaciones para el aula y para toda la institución educativa (Gráfico 7).

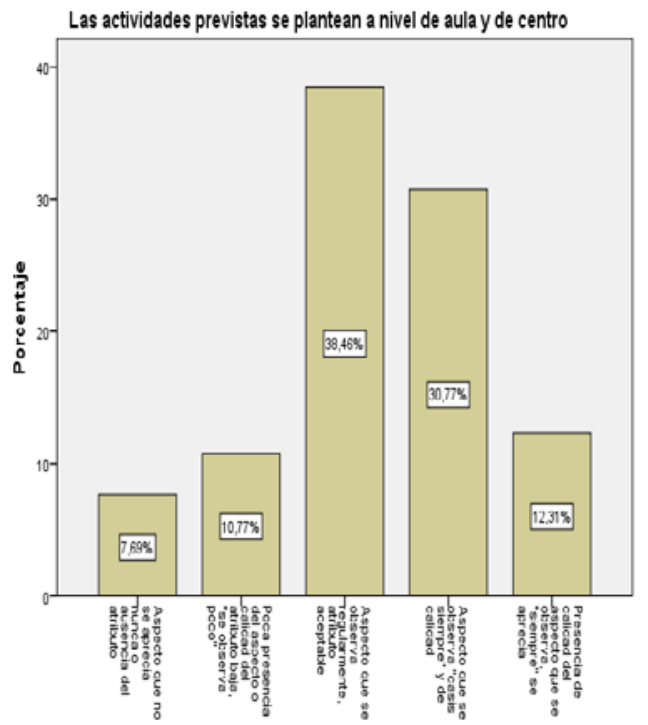

Gráfico 7. Ámbitos de las actividades

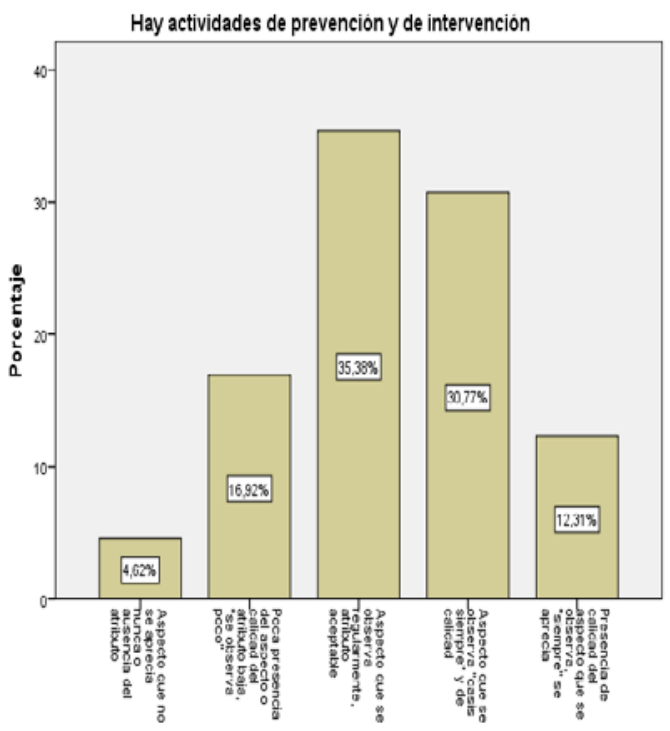

Gráfico 8. Tipología de las actividades

Un EPC manifiesta:

“Otro aspecto negativo del PC es la incoherencia entre la generalidad que hay en la gran mayoría de las actividades de convivencia frente al diagnóstico y a la especificidad que marcan los objetivos. Además, se puede ver cómo se le quiere dar importancia al ámbito familiar para poder prevenir e intervenir los problemas con los alumnos, pero los objetivos y las medidas de prevención e intervención solo van dirigidas a los alumnos" (630And).

Sobre las actividades de prevención y de intervención, en el 4.62\% de PC no existe ninguna de estas acciones; en el 16.92\% solo aparecen actividades de intervención, siendo estas mayoritariamente sanciones a los alumnos; en el 35.38\% hay acciones de intervención y son escasas las de prevención; y en el 43,08\% se incluyen especialmente acciones de prevención, pero también de intervención, aunque mayoritariamente estas últimas son sanciones (Gráfico 8).

La temporalización de las actividades, en el $40 \%$ de los PC, no se ha realizado; en el 20\%, se mencionan de forma muy genérica (ej., en el curso académico); en el 20\% se especifica la temporalización de las acciones a nivel de aula y no de centro, y solo en el $20 \%$ se plantea en todas las acciones previstas, a nivel de aula y de centro (Gráfico 9). 


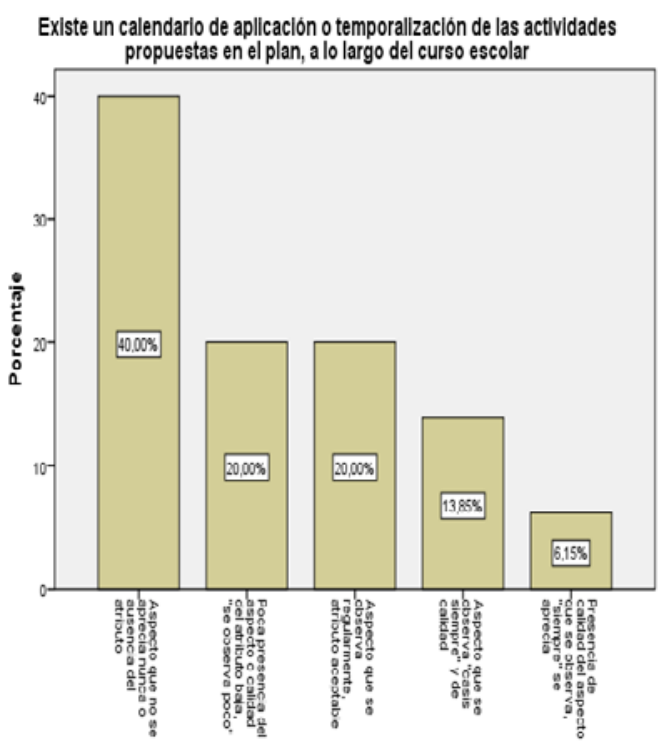

Gráfico 9. Temporalización

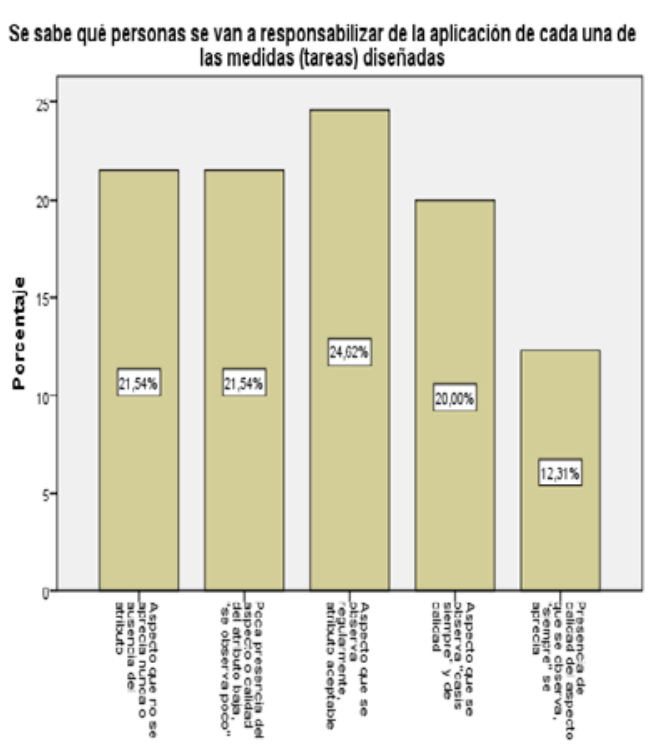

Gráfico 10. Asignación de responsables

La siguiente narrativa refleja estos hallazgos:

"En la planificación de las actividades no se especifican las fechas concretas. Se fijan plazos muy amplios, diciendo que las actividades se realizarán a lo largo del curso o de un trimestre. En algunas se puede entrever que la pretensión de ellas es el mero cumplimiento del marco legislativo, esto nos hace dudar respecto a su puesta en práctica" (215Mad).

En el 21.54\% de PC no existe ningún responsable de las actividades; en el 21.54\% la asignación es genérica (ej. el profesorado); en el 24.62\% se asignan responsables para las actividades a nivel de centro y especialmente de intervención; y solo en el 32.31\% la asignación es específica (ej. comisión de convivencia y tutor de $2^{\circ}$ ) y se realiza tanto para las acciones de prevención como de intervención (Gráfico 10). Un EPC sostiene:

"No se realiza la especificación de los responsables de cada actividad que se propone, se desconoce quiénes son los encargados de coordinarlas y/o dirigirlas, por lo que es probable que realmente no se lleven a cabo" (69And).

\section{Temática 4. Seguimiento y evaluación del plan de convivencia}

En el $27.69 \%$ de los PC no se realiza el seguimiento del plan; en el 20\% se afirma que se efectúa, pero no se indica cómo ni cuándo; en el $24.62 \%$ se señala la periodicidad 
trimestral de la evaluación, y solo en el $27.69 \%$ se indica la periodicidad, el cumplimiento de acciones y el logro de los objetivos.

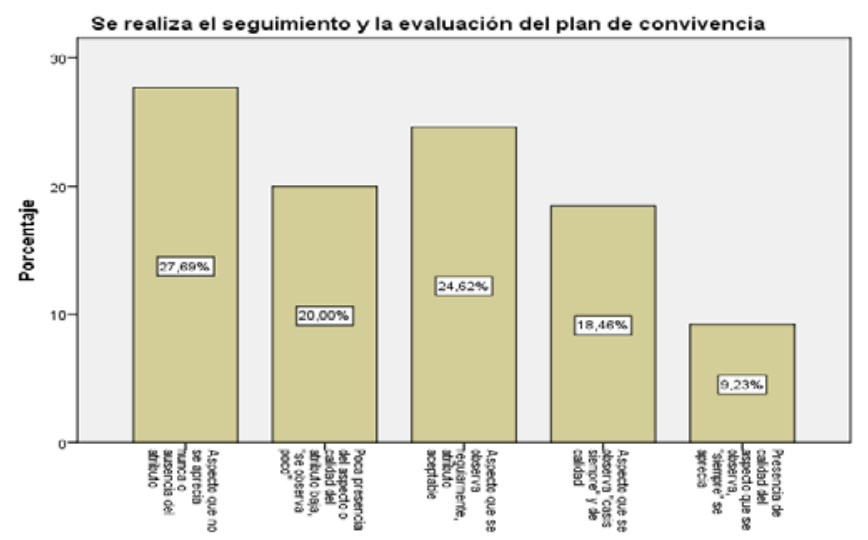

Gráfico 11. Seguimiento y evaluación del PC

La siguiente narrativa corrobora estos hallazgos:

“Este PC no lo tiene ningún tipo de seguimiento ni de evaluación. Por lo que hemos indagado, actualmente mantienen la misma versión de PC de 2009 sin ninguna modificación del documento ni de los cuestionarios y observaciones directas de distintas situaciones relacionadas con la convivencia; esto se debe a que hay una falta de seguimiento de este programa" (798Val).

\section{Discusión y conclusiones}

El objetivo de este estudio fue analizar los PC que se están implementando en los centros de Educación Secundaria en España, identificar la presencia y calidad de sus componentes, determinar sus fortalezas y debilidades, y plantear recomendaciones para un mejor diseño y desarrollo de los mismos.

Con relación al diagnóstico, se identifican dos problemas: en el $33.84 \%$ de los PC, el diagnóstico no es útil y en el $27.69 \%$ describe únicamente algunos problemas de comportamiento de los alumnos. El 26.15\% de los PC no refleja la realidad de las familias, del profesorado ni del entorno social y el $46.15 \%$ muestran una visión parcial del alumnado y de sus familias, pero no incluye la problemática del profesorado, del personal de administración y servicios ni del contexto donde se ubica el centro. De esto se colige que el diagnóstico, en el $61.53 \%$ de los casos, no contiene información útil para diseñar el PC y no permite identificar los aspectos mejorables ni definir y organizar los objetivos en función de las necesidades. No se ha indagado la realidad de la institución educativa en aspectos relacionados con la participación del alumnado en la vida del centro, los valores que el centro desea desarrollar, la igualdad, ni cómo se producen las relaciones entre iguales ni entre los miembros de la comunidad educativa; tampoco 
refleja la situación de la convivencia del entorno en el que se encuentra (72.3\%). El diagnóstico, como sostienen Conde, Azaustre y Delgado (2015), ha de ser integral y debe proporcionar una visión completa de la situación del alumnado, de sus familias y del entorno del centro, facilitando la identificación y atención de los colectivos que se encuentren en una situación de mayor vulnerabilidad (personas con discapacidad, colectivo LGBTIQ y alumnado extranjero).

Una vez que se ha reconocido la realidad, se definen los objetivos para mejorar la convivencia. Los hallazgos muestran que en el 26.15\% de los casos no existen objetivos y/o no se plantean en función del diagnóstico, y en el $40 \%$ se corresponden con el diagnóstico, incluyen a todos los miembros de la comunidad educativa, integrando especialmente a los padres, siendo esta la única fortaleza identificada en los PC. No obstante, el problema radica en que en el 30.77\% de los PC, los objetivos no son realistas, factibles, pertinentes, medibles ni son fácilmente entendibles, ya que son planteados de forma muy general y con una escasa o nula coherencia con el diagnóstico. Además, en el 29.23\% no hay objetivos vinculados con la resolución pacífica de conflictos, lo que coinciden con el estudio de Dobarro, García y Álvarez (2013) que concluyen que el alumnado percibe como menos habituales estas estrategias en sus centros educativos. Esto es preocupante ya que son conocidos sus beneficios para mejorar el clima escolar (Fernández, 2015), siendo una de las líneas de intervención más efectivas para resolver constructivamente los problemas convivenciales (Hendry, 2010; Wong, Cheng, Ngan \& Ma, 2010; Zaitegui, 2010).

En lo concerniente a las actividades, los problemas radican en que en el $49,36 \%$ de PC hay una escasa y en algunos casos nula correspondencia entre las acciones, el diagnóstico y los objetivos previstos, y se proponen acciones preventivas insuficientes para promover la participación de todos los miembros del IES. Esto es contrario a los principios que orientan el diseño y ejecución de los PC, ya que el buen clima de convivencia del centro se logra con el compromiso, acuerdo y participación de toda la comunidad educativa (Torrego \& Martínez, 2014). Tampoco es mayoritaria la presencia de instituciones educativas que planteen actividades de prevención y de intervención a nivel de aula y de centro (43.08\%), y estas últimas se proponen con el único objetivo de sancionar a los alumnos disruptivos o indisciplinados. Así señala un EPC:

"El plan tiene una normativa reguladora de la convivencia que se incluye en el RRI. Los deberes, obligaciones y castigos de los educandos están tipificados; todo se plantea desde el punto de vista sancionador; cabe incluso preguntarse si en verdad hay alguna intención real de efectuar alguna de las pocas actividades preventivas propuestas" (36 And).

La aplicación de sanciones es la actuación más formal y coactiva para gestionar la violencia escolar frente a una intervención pedagógica (Buendía, Expósito, Aguaded \& Sánchez, 2015). Concordamos con Mullet (2014) y Sharkey y Fenning (2012) en que la utilización únicamente de medidas punitivas puede sofocar la mala conducta momentáneamente; sin embargo, en el largo plazo los cambios pueden ser nulos, insignificantes o ineficaces (Kelly, 2017). En cualquier caso, ninguna perspectiva sobra por lo que se debería hablar de un modelo pedagógico integrador. 
La inconsistencia de las actividades radica la temporalización y planificación de su desarrollo. En el 40\% de los PC no existe un calendario de ejecución de las actividades y en el $20 \%$ se realiza de forma general (ej. se efectuará durante el curso académico); esto implica que en un $60 \%$ de los centros no se sabe en qué momento del curso escolar se van implementar las actividades. Asimismo, en casi en la mitad de los centros $(43.08 \%)$ se desconoce quiénes son los responsables de cada una de las tareas; esta falta de planificación y concreción de tiempo y responsables nos hace intuir que el PC es un documento meramente administrativo y formal.

Por otra parte, en el $27.69 \%$ de los PC no se realiza el seguimiento ni la evaluación y en el $20 \%$ este proceso es deficiente. Sería importante comprobar el cumplimiento de las actividades previstas de forma bimestral y no trimestral, como señala la normativa, y efectuar una evaluación anual más exhaustiva que permita verificar el logro de los objetivos para modificar el programa, actualizarlo o adaptarlo a las nuevas circunstancias.

A todas estas deficiencias, se añade la falta de actualización de los PC, ya que en muchos casos son una copia de años anteriores. Un EPC señala:

“Supuestamente el PC está actualizado y su redacción es del presente curso 2015/2016; sin embargo, hemos comprado que no es más que un copia y pega de cursos anteriores. No trata temas de actualidad tan importantes sobre los que toda la comunidad educativa debería tomar conciencia, como son el bullying, el ciberbullying, el maltrato infantil o la violencia de género" (282MAD).

Los hallazgos de la investigación también nos permiten inferir que el profesorado tiene un escaso conocimiento de recursos de prevención y tratamiento de los problemas de convivencia, lo que coincide con los resultados y conclusiones de las investigaciones de Álvarez-García et al. (2010) y González-Gil et al. (2013). Destacamos la importancia de la formación inicial y continua del profesorado en estrategias de prevención y de intervención que promuevan el conocimiento y dominio de acciones específicas para fomentar la convivencia positiva en el centro (por ej., acciones de sensibilización, educación emocional, incorporación de la convivencia como temática transversal en el currículo, formación en valores y gestión de conflictos).

En suma, la investigación nos lleva a concluir que los PC son, en general, documentos de carácter formal y burocrático, poco o nada estratégicos, ya que tienen más debilidades que fortalezas, siendo el diagnóstico, la falta o inadecuada temporalización y asignación de responsables para las actividades, y el deficiente seguimiento y evaluación los problemas más relevantes. En consecuencia, es necesario un enfoque holístico, real y práctico de la convivencia escolar, orientado al cambio en la cultura escolar.

Este estudio puede suponer una aportación a la investigación de la convivencia escolar, una guía para identificar los puntos débiles y fuertes de los PC, para replantear un diseño más realista y contextualizado, y una ejecución supervisada y controlada que permitan lograr el impacto esperado en los centros educativos y en la sociedad española.

En base a los hallazgos y conclusiones, se plantean las siguientes recomendaciones: 
- Es prioritario el cambio de rol de la administración educativa que debe pasar de ser el ente supervisor de que los centros tengan el PC a apoyar y acompañar el proceso de diseño e implementación de los PC.

- La dirección del centro ha de establecer los mecanismos apropiados, basados en el diálogo (Pradós, 2014), para que toda la comunidad educativa se involucre en las acciones previstas en el PC.

- Potenciar la formación inicial del profesorado y el desarrollo profesional docente, incorporando contenidos específicos sobre convivencia en los estudios de Grado de Magisterio y del Máster de Profesorado de Secundaria, como el aprendizaje de técnicas de mediación, negociación y resolución de conflictos, la adquisición de habilidades comunicación, competencias emocionales y el aprendizaje cooperativo.

- Realizar programas de formación específicos dirigidos a los directores y a las comisiones de convivencia de los centros.

- Desarrollar acciones de intervención y, especialmente, acciones de prevención de la violencia en los centros educativos promoviendo conductas prosociales y competencias socioemociales, previniendo comportamientos disruptivos y fomentando la resiliencia de los estudiantes y maestros (Stoiber \& Gettinger, 2011; Taub \& Pearrow, 2013).

\section{Referencias}

Álvarez-García, D., Rodríguez, C., González-Castro, P., Núñez, J. C., \& Álvarez, L. (2010). La formación en los futuros docentes frente a la violencia escolar. Revista de Psicodidáctica, 15(1), 35-56.

Álvarez-García, D., Dobarro, A., Rodríguez, C., Núñez, J. C., \& Álvarez, L. (2013). El consenso de normas de aula y su relación con bajos niveles de violencia escolar. Infancia y Aprendizaje, 36(2), 199-217. doi: https://doi.org/10.1174/021037013806196229

Boqué, M. C. (2002). Guía de mediación escolar. Programa comprensivo de actividades, etapas primaria y secundaria. Barcelona: Octaedro.

Buendía, L., Expósito, J., Aguaded, E., \& Sánchez, C. (2015). Análisis de la convivencia escolar en las aulas multiculturales de Educación Secundaria. Revista de Investigación Educativa, 33(2), 303-319. doi: https://doi.org/10.6018/rie.33.2.211491

Burton, B. (2012). Peer teaching as a strategy for conflict management and student reengagement in schools. Australian Educational Researcher, 39, 45-58. doi: https://eric. ed.gov/?redir=http\%3a\%2f\%2fdx.doi.org\%2f10.1007\%2fs13384-011-0046-4

Carbonell, J. L. (Coord.) (1997). Convivir es vivir. Madrid: Defensor del Menor.

Cerezo, F. (2011). Políticas de convivencia escolar: percepción y eficacia desde la perspectiva familiar. Revista Electrónica Interuniversitaria de Formación del Profesorado, 14(1), 313-323.

Conde, S., Azaustre, C., \& Delgado, M. (2015). Análisis integral de la gestión de la convivencia escolar. Una propuesta de evaluación. International Journal for 21st Century Education, 2(1), 39-59. doi: https://doi.org/10.21071/ij21ce.v2i1.4260

De Miguel, M. (2009). Evaluación de planes y programas de ciudadanía y convivencia. Praxis, 13, 47-69. 
Díaz-Aguado, M. J., Segura, M. P., Royo, P., \& Andrés, M. (1996). Programas de educación para la Tolerancia y prevención de la violencia en los jóvenes. Instrumentos de evaluación e investigación (Vol. IV). Madrid: Ministerio de Trabajo y Asuntos Sociales.

Dobarro, A., García, T., \& Álvarez, D. (2013). ¿Qué medidas para la mejora de la convivencia se están desarrollando en los centros educativos?: una perspectiva desde dentro. European Journal of Investigation in Health, Psychology and Education, 3(3), 207-213. doi: https://doi.org/10.30552/ejihpe.v3i3.50

Fernández, J. M. (2015). Convivencia escolar. Un estudio en la provincia de Sevilla (España). International Journal of Educational Research and Innovation, 3, 78-93.

Gázquez, J. J., Pérez-Fuentes, M. C., Lucas, F., \& Fernández, M. (2011). Análisis de la convivencia escolar por los docentes europeos. Aula Abierta, 39(1), 51-58.

Gil, F., Delgado, M. A., \& Chillón, P. (2016). Percepción de las conductas contrarias a la convivencia en el profesorado de Educación Secundaria Obligatoria. SPORT TK-Revista EuroAmericana de Ciencias del Deporte, 5(2), 167-178. doi: https://doi.org/10.6018/264821

González-Gil, F., Martín-Pastor, E., Flores, N., Jenaro, C., Poy, R., \& Gómez-Vela, M. (2013). Inclusión y convivencia escolar: análisis de la formación del profesorado. European Journal of Investigation in Health, Psychology and Education, 3(2), 125-135. doi: https://doi.org/10.30552/ejihpe.v3i2.41

Hendry, J. (2010). Mediation in schools: Tapping the potential. International Journal of School Disaffection, 1, 26-32.

Jares, X. (1997). El lugar del conflicto en la organización escolar. Revista Iberoamericana de Educación, 15, 53-73.

Kelly, D. R. (2017). Methods for reducing violence in schools: A systematic review. Journal of Educational and Developmental Psychology, 7(1), 200-209. doi: https://doi. org/10.5539/jedp.v7n1p200

Ley Orgánica 2/2006, de 3 de mayo, de Educación. Boletín Oficial del Estado 106, de 04/05/2006.

Ley Orgánica 8/2013, de 9 de diciembre, para la Mejora de la Calidad Educativa. Boletín Oficial del Estado 295, de 10 de diciembre de 2013.

Mullet, J. H. (2014). Restorative discipline: From getting even to getting well. Children $\mathcal{E}$ Schools, 36(3), 157-162. doi: https://doi.org/10.1093/cs/cdu011

Musitu, G., Suárez, C., Del Moral, G., \& Villareal, M. E. (2015). El consumo de alcohol en adolescentes: el rol de la comunicación, el funcionamiento familiar, la autoestima y el consumo en la familia y amigos. Búsqueda, 14, 45-61. doi: https://doi. org/10.21892/01239813.58

Ortega, R. (1997a). El proyecto Sevilla anti-violencia escolar. Un modelo de intervención preventiva contra los malos tratos entre iguales. Revista de Educación, 313, 143-161.

Ortega, R. (1997b). ¿Es la violencia un problema emergente en la infancia? En J. A. Ponce, R. Muriel, \& I. Gómez de Terreros (Eds.), Informe salud, infancia, adolescencia y sociedad. Sección de pediatría social (pp. 76-82). Sevilla: Asociación Española de Pediatría.

Ortega R., \& Del Rey, R. (2001). Aciertos y desaciertos del Proyecto Sevilla anti-violencia escolar (SAVE). Revista de Educación, 324, 253-270.

Ortega, R., Del Rey, R., \& Casas, J. A. (2014). La convivencia escolar: clave en la predicción del bullying. Revista Iberoamericana de Evaluación Educativa, 6(2), 91-102.

Osterlind, S. J. (1989). Constructing test items. Boston: Kluwer Academic Publishers. 
Padrós, M. (2014). A transformative approach to prevent peer violence in schools: contributions from communicative research methods. Qualitative Inquiry, 20(7), 916-922. doi: https://doi.org/10.1177\%2F1077800414537217

Penalva, A., Hernández, M. A., \& Guerrero, C. (2014). Percepción de los expertos de la necesidad de la formación del profesorado en convivencia. Revista Fuentes, (15), 281-304. doi: http://dx.doi.org/10.12795/revistafuentes.2014.i15.13

Pérez-Fuentes, M., Álvarez-Bermejo, J. A., Molero, M., Gázquez, J. J., \& Vicente, M. (2011). Violencia escolar y rendimiento académico (VERA): aplicación de realidad aumentada. European Journal of Investigation in Health, Psychology and Education, 1(2), 71-84. doi: https://doi.org/10.30552/ejihpe.v1i2.19

Piñero, E., Arense, J. J., López, J. J., \& Torres, A. M. (2014). Incidencia de la violencia y victimización escolar en estudiantes de Educación Secundaria Obligatoria en la Región de Murcia. Revista de Investigación Educativa, 32(1), 223-241. doi: https://doi. org/10.6018/rie.32.1.154251

Sabariego, M. (2012). La investigación educativa: génesis, evolución y características. En. R. Bisquerra (Coord.), Metodología de la investigación educativa (pp. 51-87). Madrid: La Muralla.

Sharkey, J. D., \& Fenning, P. A. (2012). Rationale for designing school contexts in support of proactive discipline. Journal of School Violence, 11(2), 95-104. doi: https://doi. org/10.1080/15388220.2012.646641

Stoiber, K. C., \& Gettinger, M. (2011). Functional assessment and positive support strategies for promoting resilience: Effects on teachers and high-risk children. Psychology in the Schools, 48, 686-706. doi: https://doi.org/10.1002/pits.20587

Stufflebeam, D. (2001). The metaevaluation imperative. American Journal of Evaluation, 22(2), 183-209. doi: https://doi.org/10.1177\%2F109821400102200204

Taub, J., \& Pearrow, M. (2013). Resilience through violence and bullying prevention in schools. In S. Goldstein, \& R. B. Brooks (Eds.), Handbook of resilience in children (pp. 371-386). Boston: Springer US.

Torrego, J. C. (Coord.). (2006). Modelo integrado de mejora de la convivencia. Barcelona: Graó.

Torrego, J. C., \& Martínez, C. (2014). Claves para el desarrollo del Plan de Convivencia en los centros educativos desde una perspectiva integral. Qualitative Research in Education, 3(1), 83-113. doi: http://dx.doi.org/10.4471/qre.2014.37

Trianes, M. V., \& Fernández-Figarés, C. (2001). Aprender a ser personas y a convivir. Un programa para Secundaria. Bilbao: Descleé de Brouwer.

Wong, D. S. W., Cheng, C. H.K., Ngan, R. M. H., \& Ma, S.K. (2010) Program effectiveness of a restorative whole-school approach for tackling school bullying in Hong Kong. International Journal of Offender Therapy and Comparative Criminology, 55(6), 846-862. doi: https://doi.org/10.1177\%2F0306624X10374638

Zaitegi, N. (2010). La educación en y para la convivencia positiva en España. REICE. Revista Iberoamericana sobre Calidad, Eficacia y Cambio en Educación, 8(2), 93-132.

Fecha de recepción: 5 de diciembre de 2017.

Fecha de revisión: 11 de diciembre de de 2017.

Fecha de aceptación: 8 de marzo de 2019. 
